\title{
Near-Fatal Angioedema Compromising the Airway in a Patient Following Angiotensin Converting Enzyme Inhibitor Formulary Substitution
}

\author{
John T. Denny ${ }^{\mathrm{a}, \mathrm{c}}$, Angela M. Denny ${ }^{\mathrm{b}}$, Harris Shaikh ${ }^{\mathrm{a}}$, Sana Shaikh ${ }^{\mathrm{a}}$, Mohammad Chaudhry ${ }^{\mathrm{a}}$
}

\begin{abstract}
We present an unusual case of life-threatening angioedema (AE) with airway compromise that occurred after a formulary substitution of one angiotensin converting enzyme inhibitor (ACEI) for another. $\mathrm{AE}$ is a known side effect of ACEI therapy. We report a rare case in which a patient who had tolerated years of therapy with enalapril, developed life-threatening AE shortly after being switched to another ACEI: ramipril. AE is of particular importance in airway management as it may present at any point in the emergency ward or hospital stay and it may rapidly become life-threatening if it involves airway compromise. Patient was an 81-year-old female with a past history including peripheral vascular disease, hypertension, and congestive heart failure. Patient was in the status of post femoral artery endarterectomy which subsequently became infected and required an incision and drainage, and subsequent flap closure. The patient was extubated post-operatively. The two main etiologies of AE are mast cell-mediated or kinin-related, although there are less frequent types as well. About $0.1-2.2 \%$ of patients treated with ACEI develop recurrent AE, often facial or lip swelling or edema of the tongue and/or larynx. The degree of swelling can be severe: cases of death by asphyxiation following closure of the upper airways have been reported. The interval between starting the ACEI and the appearance of the first AE can be months or even several years, so that making the linkage between the $\mathrm{AE}$ and the triggering ACEIs is sometimes unrecognized or recognized late. Because of the very common use of ACEIs, cases of AE are not rare. $\mathrm{AE}$ is a medical emergency and requires close monitoring as is routinely provided in an intensive care unit. It is important for practitioners to recognize that $\mathrm{AE}$ patients likely have altered airway anatomy and will be difficult intubations. It is crucial to then choose to not sedate or induce unconsciousness prior to securing the airway in these patients. Anticipating difficulty in one's ability to readily secure an airway requires the presence of experienced airway profes-
\end{abstract}

Manuscript accepted for publication December 04, 2014

aDepartment of Anesthesia, Rutgers/Robert Wood Johnson Medical School, New Brunswick, NJ, USA

${ }^{b}$ Rutgers Graduate School of Nursing, Newark, NJ, USA

${ }^{\mathrm{c} C o r r e s p o n d i n g ~ A u t h o r: ~ J o h n ~ T . ~ D e n n y, ~ D e p a r t m e n t ~ o f ~ A n e s t h e s i a, ~ R u t g e r s / ~}$ Robert Wood Johnson Medical School, 3100 CAB, 125 Paterson Street, New Brunswick, NJ 08901, USA. Email: dennyjt@rwjms.rutgers.edu

doi: http://dx.doi.org/10.14740/jmc2019w sionals and the ready availability of specialized equipment.

Keywords: Angioedema; Airway compromise; Angiotensin converting enzyme inhibitors

\section{Introduction}

We present an unusual case of life-threatening angioedema (AE) with airway compromise that occurred after a formulary substitution of one angiotensin converting enzyme inhibitor (ACEI) for another, in a patient on long-term ACEI. AE is short-lived edema in the deep dermal layer of the skin and the subcutaneous tissue. Similar edema episodes may occur in the submucosal tissue of various organs, such as the larynx or bowel [1].

In $\mathrm{AE}$, the local plasma extravasates to the interstitium resulting in non-pitting, nondependent, and often edema that is asymmetric [2]. It usually develops quickly over minutes, rarely occurs over hours, and most often resolves in less than $48 \mathrm{~h}$. Areas affected primarily include the mucosa and skin of the face, throat, larynx, bowel, genitalia, and extremities. It is felt that subcutaneous or submucosal capillaries and postcapillary venules develop increased permeability as a result of vasoactive mediators such as bradykinin, histamine, complement, prostaglandin $\mathrm{D} 2$, and proteases [1]. AE is of particular importance in airway management as it may present at any point in the emergency ward or hospital stay and it may rapidly become life-threatening if it involves airway compromise. This is especially true because of the tendency for tongue and laryngeal involvement in swelling.

\section{Case Report}

$\mathrm{AE}$ is a known side effect of ACEI therapy. We report a rare case in which a patient, who had tolerated years of therapy with enalapril, developed life-threatening $\mathrm{AE}$ after being switched to ramipril in the hospital. Patient was an 81-yearold female with a past history of peripheral vascular disease, diabetes mellitus, hypertension, congestive heart failure, status post coronary artery bypass grafting, atrial fibrillation, and transient ischemic attack. Patient was in the status of post fem- 
oral artery endarterectomy which subsequently became infected and required an incision and drainage and subsequent flap closure. The patient was extubated post-operatively. Her home anti-hypertensive medication of enalapril was substituted with another ACEI ramipril. Six hours later, patient developed AE with stridor. Patient was initially treated with nebulized epinephrine, benadryl and hydrocortisone, with only partial response.

With the tongue swelling, the patient was correctly identified as a "difficult airway" (DA), and anesthesia was called immediately to evaluate the patient. Since the patient's respiratory status was deteriorating but still temporarily adequate, and likely a DA, an awake endotracheal intubation was chosen. The awake intubation technique is well suited for a patient with a recognized difficult airway. An awake technique has a number of advantages: it preserves spontaneous ventilation by the patient, rather than pharmacologically depressing respiration. Spontaneous respiration also preserves muscle tone which greatly aids in maintaining an open airway. A glidescope was used to advance a 6.0 ET tube after two previous attempts failed due to airway edema. Patient breathed spontaneously during the awake intubation. Pulse oximetry remained greater than $98 \%$ and vital signs were stable. After intubation, patient was started on a steroid taper and weaned. She was extubated uneventfully 3 days later. She remained successfully extubated.

\section{Discussion}

The renin angiotensin system (RAS) is the body's most powerful hormone system for controlling renal hemodynamics and the excretion of sodium and, therefore, body fluid volumes and arterial pressure. The discovery of ACEI was central to the understanding of the significance of the RAS since ACEI proved to be effective in controlling hypertension, treating heart failure and in preventing the development of the vascular injury of chronic diseases like diabetes mellitus. The success of ACEI stimulated research into inhibitors of other actors of the RAS like renin or angiotensin receptor antagonists. Basic research was the key to the discovery of ACEI: after the observation that the prey of the Brazilian Pit Viper (Bothrops Jararaca) became edematous and suddenly hypotensive. Examination of the snake's prey found increased levels of bradykinin and evaluation of the venom led to the discovery of peptides that inhibit angiotensin I conversion to angiotensin II [3].

The two main etiologies of AE are mast cell-mediated or kinin-related, although there are less frequent types as well.

About $0.1-2.2 \%$ of patients treated with ACEIs develop recurrent $\mathrm{AE}$, often facial or lip swelling or edema of the tongue and/or larynx (AE induced by ACEIs, AE-ACEI) [4, 5]. The degree of swelling can be severe: cases of death by asphyxiation following closure of the upper airways have been reported [6]. The interval between starting the medication and the appearance of the first AE can be months or even several years, so that making the linkage between the AE and the triggering ACEIs is sometimes unrecognized or recognized late. Because of the very commonuse of ACEIs, cases of AE-ACEI are not rare. Angiotensin II receptor blocker medications can also trigger the same forms of AE, though more rarely [1]. Occasionally chronic urticaria is associated with AE-ACEI.

Several risk factors can predispose to AE, such as female sex and being African-American. Both these risk factors were present in our patient. Most cases occur in the first week to 90 days of treatment, although sometimes symptom onset may be delayed for years [7]. Less well-known than the airway compromise is that gastrointestinal tract AE also occurs and typically presents as abdominal pain accompanied by nausea, vomiting, and/or diarrhea. The mechanism of gastrointestinal $\mathrm{AE}$ is vasodilation and increased vascular permeability as a result of increased levels of bradykinin [8]. Benson et al report a case series of intermittent abdominal symptoms in patients due to ACEIs [9].

\section{Conclusions}

$\mathrm{AE}$ is a medical emergency and requires close monitoring as is routinely provided in an intensive care unit. It is important for practitioners to recognize that $\mathrm{AE}$ patients likely have altered airway anatomy and will be difficult intubations. It is crucial to then choose to not sedate or induce unconsciousness prior to securing the airway in these patients. Anticipating difficulty in one's ability to readily secure an airway requires the presence of experienced airway professionals and the ready availability of specialized equipment that will facilitate adherence to the American Society of Anesthesiologist's algorithm for DA management.

Rendering this particular patient unconscious with drugs would likely have produced a catastrophic situation where the patient could not be easily intubated, nor could be mask ventilated. This cannot ventilate, cannot intubate situation can rapidly progress to desaturation and cardiac arrest. This is especially true in intensive care unit patients (even those with normal airways) who commonly have very limited reserves. ACEI can cause AE even after prolonged use. In this case, an acute change in the ACEI prescribed likely triggered the AE. It may be appropriate to be cautious in switching ACEIs without a compelling reason.

\section{References}

1. Bork K. Angioedema. Immunol Allergy Clin North Am. 2014;34(1):23-31.

2. Kulthanan K, Jiamton S, Boochangkool K, Jongjarearnprasert K. Angioedema: clinical and etiological aspects. Clin Dev Immunol. 2007;2007:26438.

3. Ferreira SH. Angiotensin converting enzyme: history and relevance. Semin Perinatol. 2000;24(1):7-10.

4. Byrd JB, Adam A, Brown NJ. Angiotensin-converting enzyme inhibitor-associated angioedema. Immunol Allergy Clin North Am. 2006;26(4):725-737.

5. Sabroe RA, Black AK. Angiotensin-converting enzyme (ACE) inhibitors and angio-oedema. Br J Dermatol. 1997;136(2):153-158. 
6. Dean DE, Schultz DL, Powers RH. Asphyxia due to angiotensin converting enzyme (ACE) inhibitor mediated angioedema of the tongue during the treatment of hypertensive heart disease. J Forensic Sci. 2001;46(5):12391243.

7. Campo P, Fernandez TD, Canto G, Mayorga C. Angioedema induced by angiotensin-converting enzyme inhibitors. Curr Opin Allergy Clin Immunol. 2013;13(4):337-344.
8. Cicardi M, Johnston DT. Hereditary and acquired complement component 1 esterase inhibitor deficiency: a review for the hematologist. Acta Haematol. 2012;127(4):208220.

9. Benson BC, Smith C, Laczek JT. Angiotensin converting enzyme inhibitor-induced gastrointestinal angioedema: a case series and literature review. J Clin Gastroenterol. 2013;47(10):844-849. 